\title{
Radio Range Adjustment for Energy Efficient Wireless Sensor Networks
}

\author{
Q. Gao ${ }^{\text {a, }}$, K. J. Blow ${ }^{\text {a } 1}$, D. J. Holding ${ }^{\text {a }}$, I. W. Marshall ${ }^{\text {b }, ~ X . ~ H . ~ P e n g ~}{ }^{\text {a }}$ \\ ${ }^{\text {a }}$ Electronic Engineering, Aston University, Birmingham B4 7ET,United Kingdom

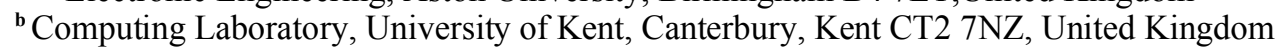

\begin{abstract}
In wireless ad hoc sensor networks, energy use is in many cases the most important constraint since it corresponds directly to operational lifetime. Topology management schemes such as GAF put the redundant nodes for routing to sleep in order to save the energy. The radio range will affect the number of neighbouring nodes, which collaborate to forward data to a base station or sink. In this paper we study a simple linear network and deduce the relationship between optimal radio range and traffic. We find that half of the power can be saved if the radio range is adjusted appropriately compared with the best case where equal radio ranges are used.
\end{abstract}

Keywords: Sensor network; energy saving; range adjustment

\section{Introduction}

Recent advances in micro-electro-mechanical systems (MEMS) technology, wireless communications and digital electronics have enabled the development of low-cost, low-power, multifunctional smart sensor nodes. Smart sensor nodes are autonomous devices equipped with heavily integrated sensing, processing, and communication capabilities. When these nodes are networked together in an ad-hoc fashion, they form a sensor network. The nodes gather data via their sensors, process it locally or coordinate amongst neighbors and forward the information to the user or, in general, a data sink.

Since these integrated sensor nodes have highly compact form factors and are wireless, they are highly energy constrained. Furthermore, replenishing energy via replacing batteries on up to thousands of nodes (in possibly harsh terrain) is infeasible. Hence, it is well accepted that one of the key challenges in unlocking the potential of such data gathering sensor networks is conserving energy so as to maximize their post-deployment active lifetime. In terms of energy consumption, the wireless exchange of data between nodes strongly dominates other node functions such as sensing and processing. Moreover, actual radios consume power not only when sending and receiving data, but also when listening. Stemm and Katz show idle:receive:transmit ratios are 1:1.05:1.4 by measurement [1], while more recent studies show ratios of 1:2:2.5 [2] and 1:1.2:1.7[3]. Significant energy savings are only obtainable by putting as many nodes as possible to sleep.

Topology management provides the distributed resources to the overlying applications in an energy efficient manner to achieve the service requirements for the maximum possible time. Taking advantage of high-density deployment, each node can assess its connectivity and adapts its participation in the multi-hop network topology based on local measurements to extend overall system lifetime. If we increase the radio range there are more nodes in the collaborative area, which can decide to go to sleep and therefore prolong the network's lifetime. So far, we have assumed that the energy demanded for transmission is independent of the distance. In fact, airborne radio transmissions are attenuated by a path loss in a power-law with distance. Since the path loss of radio transmission scales with distance in a greater-than-linear fashion [11], the total transmission energy can be reduced by dividing a long transmission path into several shorter ones. Now the problem is how can we reach the optimal range for energy efficient routing that uses the smallest amount of energy for data transmission while simultaneously allowing many nodes to be put into the sleep state. In this paper we deduce the relationship between optimal radio range and traffic and find that half of the power can be saved if the radio range is adjusted according to the optimum strategy in a linear network compared with the best case where equal radio ranges are used.

\section{Radio Power Model and Characteristic Distance}

For a simplified power model of radio communication [4][5], the energy consumed per second in transmission is:

\footnotetext{
${ }^{1}$ Corresponding author. Tel.: +44-0121-3596987; fax: +44-0121-3590156.

E-mail addresses: qianggao@aston.ac.uk (Q. Gao), k.j.blow@aston.ac.uk (K. J. Blow), holdindj@aston.ac.uk (D. J. Holding), i.w.marshall@kent.ac.uk (I. W. Marshall),x-h.peng@aston.ac.uk (X. H. Peng)
} 


$$
E_{t}=\left(e_{t}+e_{d} r^{n}\right) B
$$

where $e_{t}$ is the energy/bit consumed by the transmitter electronics (including energy costs of imperfect duty cycling due to finite startup time), and $e_{d}$ accounts for energy dissipated in the transmit op-amp (including op-amp inefficiencies). Both $e_{t}$ and $e_{d}$ are properties of the transceiver used by the nodes, $r$ is the transmission range used. The parameter $n$ is the power index for the channel path loss of the antenna. This factor depends on the RF environment and is generally between 2 and 4 . $B$ is the bit rate of the radio and is a fixed parameter in our study.

On the receiving side, a fixed amount of power is required to capture the incoming radio signal

$$
E_{r}=e_{r} B
$$

where $e_{r}$ is the energy/bit consumed by the receiver electronics used by the node. Typical numbers for currently available radio tranceivers are $e_{t}=50 \times 10^{-9} \mathrm{~J} / \mathrm{bit}, e_{r}=50 \times 10^{-9} \mathrm{~J} / \mathrm{bit}, e_{d}=100 \times 10^{-12} \mathrm{~J} / \mathrm{bit} / \mathrm{m}^{2}$ (for $\mathrm{n}=2)$ and $B=1 \mathrm{Mbit} / \mathrm{s}[6]$.

Since the path loss of radio transmission scales with distance in a greater-than-linear fashion, the transmission energy can be reduced by dividing a long path into several shorter ones. However, if the number of intermediate nodes is very large then the energy consumption per node is dominated by the term $e_{t}$ in equation (1) and the receiving energy consumption hence an optimum exists. Intermediate nodes between a data source and destination can serve as relays that receive and rebroadcast data

Let us consider multihop communication in a finite one dimensional network from the source to the base station across a distance $d$ using $k$ hops. The source at $x=d$ will generate traffic of $A$ Erlang, so that each intermediate node receives and transmits the same traffic, $A$. The routing nodes are assumed to be regularly spaced and to consume no energy while idle. The power consumed by this communication is then simply the sum of the transmit and receive energies multiplied by the effective bit rate, $B A$, and is given by

$$
P=\sum_{i=1}^{k}\left(e_{t}+e_{d} r_{i^{n}}+e_{r}\right) B \quad A, \sum_{i=1}^{k} r_{i}=d
$$

In order to minimize $P$ we note that it is strictly convex and use Jensen's inequality. Given $d$ and $k$ then $P$ is minimized when all the hop distances $r_{i}$ are made equal to $d / k$. The minimum energy consumption for a given distance $d$ has either no intervening hops or $k_{\text {opt }}$ equidistant hops where $k_{\text {opt }}$ is always one of,

$$
k_{\text {opt }}=\left(\frac{d}{d_{\text {char }}}\right) \text { or } k_{\text {opt }}=\left(\frac{d}{d_{\text {char }}}\right)
$$

The distance $d_{c h a r}$, called the characteristic distance, is independent of $d$ and is given by,

$$
d_{\text {char }}=\sqrt[n]{\frac{e_{t}+e_{r}}{e_{d}(n-1)}}
$$

The characteristic distance depends only on the energy consumption of the hardware and the path loss coefficient (i.e. it is independent of the traffic); $d_{\text {char }}$ alone determines the optimal number of hops. For typical COTS (commercial, off-the-shelf)-based sensor nodes, $d_{c h a r}$ is about 35 meters. The introduction of relay nodes is clearly a balancing act between reduced transmission energy and increased receive energy. Hops that are too short lead to excessive receive energy. Hops that are too long lead to excessive path loss. In between these extremes is an optimum transmission distance that is the characteristic distance.

\section{Topology Management}

The traffic distribution through appropriate routing essentially exploits the macro-scale redundancy of possible routes between source and destination. However, on each route, there is also a micro-scale redundancy of nodes that are essentially equivalent for the multi-hop path. In typical deployment scenarios, a dense network is required to ensure adequate coverage of both the sensing and multi-hop routing functionality, in addition to improving network fault-tolerance. Despite the inherent node redundancy, these high densities do not immediately result in an increased network lifetime, as the radio energy consumption in idle mode does not differ much from that in transmit or receive mode. Only transitioning the radio into the sleep state can temporarily quiescent nodes to conserve battery energy. However, in this state, nodes cannot be communicated with, and have effectively retracted from the network, thereby changing the active topology. Thus, the crucial issue is to intelligently manage the sleep state transitions while maintaining robust undisturbed operation.

This reasoning is the foundation for topology management approach, which explicitly leverages the fact that in high node density several nodes can be considered backups of each other with respect to 
traffic forwarding. Achieving energy saving through activation of a limited subset of nodes in an ad-hoc wireless network has also been the goal of some recent research such as GAF [7], SPAN [3], ASCENT [8], CEC [9] and AFECA [10].

In SPAN, a limited set of nodes forms a multi-hop forwarding backbone, which tries to preserve the original capacity of the underlying ad-hoc network. Other nodes transition to sleep states more frequently, as they no longer carry the burden of forwarding data of other nodes. To balance out energy consumption, the backbone functionality is rotated between nodes and therefore there is a strong interaction with the routing layer.

In ASCENT, the decision for being active is the courtesy of the node. Passive nodes keep listening all the time and assess their course of actions; stay passive or become active.

Cluster-based Energy Conservation (CEC) and the Adaptive Fidelity Energy-Conserving Algorithm (AFECA) are two other proposed energy conserving topology management algorithms. CEC creates clusters and selects cluster-heads based on the highest advertised remaining energy. AFECA allows each node to sleep for randomized periods based on the number of (overheard) neighbors it has.

The GAF algorithm is based on a division of the sensor network in a number of virtual grids of size $R$ by $R$, see Figure 1 . The value of $R$ is chosen such that all nodes in a grid are equivalent from a routing perspective. This means that any two nodes in adjacent grids should be able to communicate with each other. By investigating the worst-case node locations depicted in Figure 1, we can calculate that $R$ should satisfy

$$
R \leq \frac{r}{\sqrt{5}}
$$

For the one dimension case, $R$ should satisfy

$$
R \leq r / 2
$$

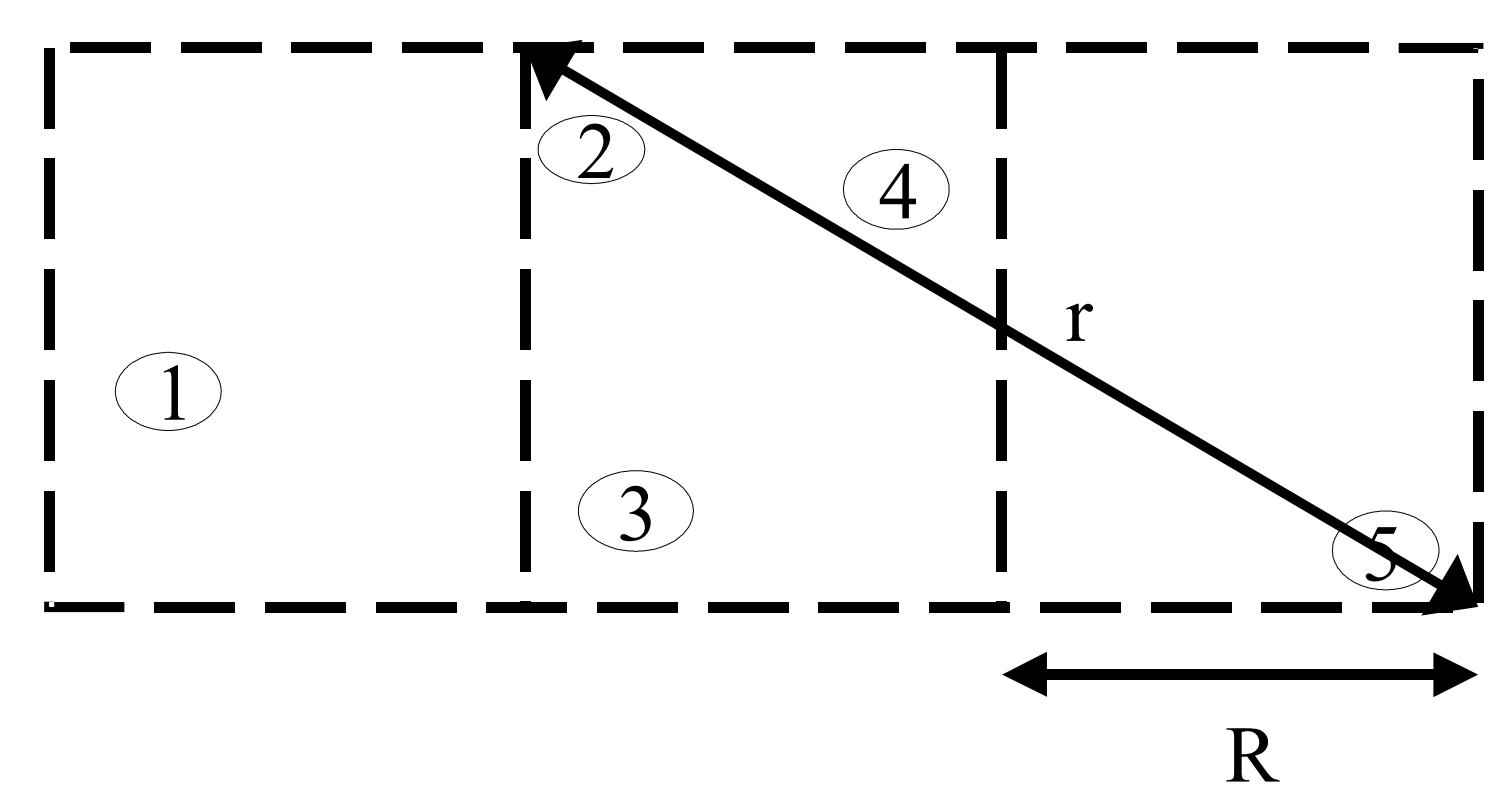

Figure 1: GAF virtual grid structure

Since all nodes in a grid are equivalent from a routing perspective, we can use this redundancy to increase the network lifetime. GAF only keeps one node awake in each grid, while the other nodes put their radio in the sleep mode. To balance out the energy consumption, the burden of traffic forwarding is rotated between nodes. For simplicity, we ignore the unavoidable time overlap of this process associated with handoff. If there are $m$ nodes in a grid, the node will (ideally) only turn its radio on for a fraction $1 / m$ of the time and therefore will last $m$ times longer. If we increase the radio transmission range, $r$, there will be more nodes within each grid and hence more redundant nodes can make the transition into the sleep state and therefore a longer network lifetime can be achieved. Since the energy consumption associated with transmission increases super-linearly with radio range, there will be an optimum range that provides the maximum energy saving.

Topology management algorithms work well in high density sensor networks. They let redundant nodes go to sleep and network life is prolonged while the connection and capacity of the networks are 
preserved. For GAF there are many nodes in a grid section when densities are high and long lifetime can be achieved. Furthermore in high-density networks if some nodes are transition into sleep state, collisions can be reduced when several neighboring nodes compete to access the transmission medium. And overhearing energy waste can be reduced as well for topology management strategy.

\section{Relationship between Range and Traffic}

In section 2 we introduced a simple energy model in which no energy was consumed while the node was idle. This led to a characteristic distance that was independent of traffic. We now include the idle state energy and show how the characteristic distance is modified. On one hand a short range is preferred for energy efficient data transmission as a result of the nonlinear path loss ratio. On the other hand more redundant nodes can be put into the sleep state to prolong the network lifetime if a long range is used in the topology management of sensor networks. So what is the optimal range from an energy efficiency perspective? Again, we consider a linear network of length $d$ in which the traffic carried from end to end is $A$ Erlang. If the transmission route is divided into $k$ grids and only one node wakes up in each grid as relay node, as in the GAF protocol, the total energy consumption per second by $k$ hops is

$$
P=k\left[e_{r} B A+e_{t} B A+e_{d}\left(2 \frac{d}{k}\right)^{n} B A+c e_{r}(1-2 A) B\right]
$$

The last term $c e_{r}(1-2 A) B$ in the equation (8) represents the energy consumption when the radio neither receives nor transmits, i.e. it is in the idle state. The energy consumption in the idle state is approximately equal to that in the receiving state, so that the parameter $c$ is close to 1 . Note that we are currently assuming that nodes in the sleep state consume no energy. Also, we assume that the routing node in each grid can be located anywhere within that section and so the radio range is now twice the grid size. The energy efficient optimum size of the virtual grid can now be derived from equation (8) and is given by

$$
R_{\text {opt }}=r_{\text {opt }} / 2=\sqrt[n]{\frac{\left(e_{r}+e_{t}\right) A+c e_{r}(1-2 A)}{2^{n} A(n-1) e_{d}}}
$$

The minimum energy consumption characteristic range is no longer a constant and changes with the amount of traffic. Figure 2 shows the relationship between the traffic $A$ and the optimal range $r_{\text {opt }}$. The optimal range is decreasing as the loaded traffic increases. At the extreme point $A=0.5$, where the transmitter spends $50 \%$ of the time transmitting and $50 \%$ receiving (we assume the node can only do one or the other), there is no idle time and so the optimal range converges to $d_{\text {char }}$. Under conditions of light traffic the optimal range increases sharply as the loaded traffic decreases. When the data transferred in the sensor network is low, the idle state dominates the energy consumption and hence the radio range can be relatively large.

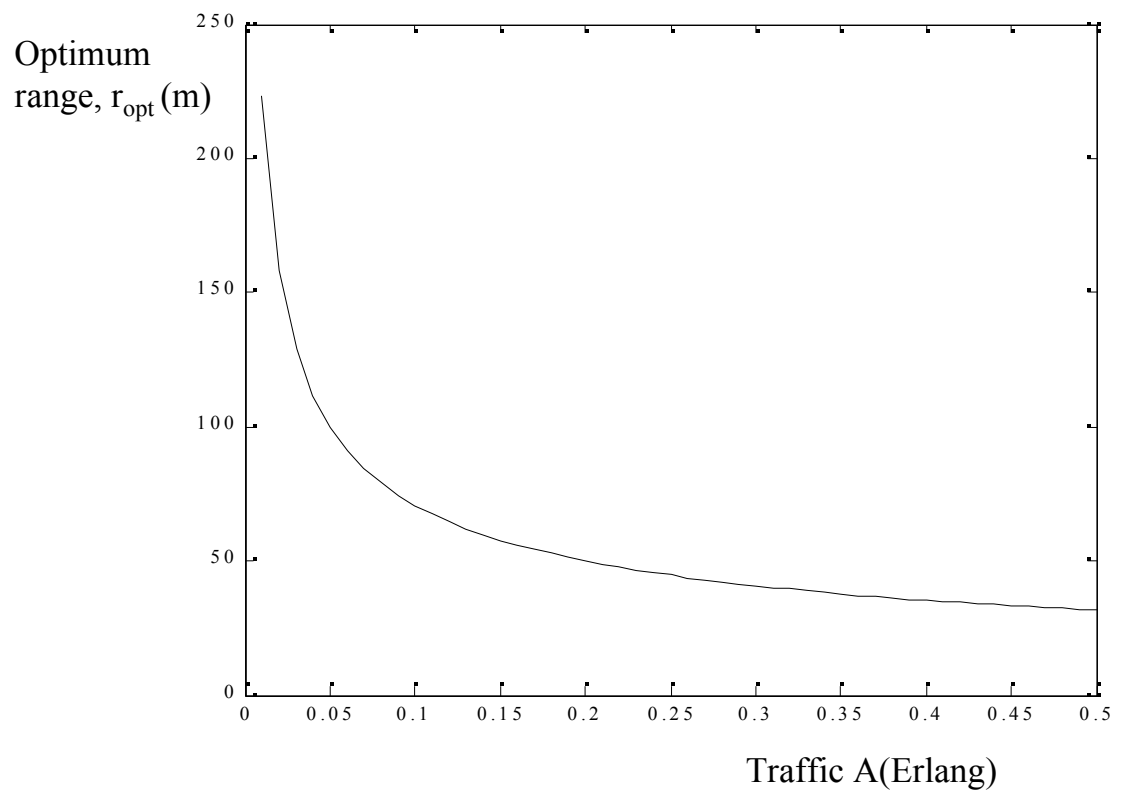


Figure 2: Optimum radio range as a function of the network traffic

\section{Transmission Range Adjustment}

So far, we have only considered data in transit across a linear network of routing nodes. In a real sensor network, the data are generated internally. In many applications of wireless sensor networks, data is gathered by multiple sensors at different locations and transmitted to a single sink node (such as a base station) where data can be stored and analyzed. If the relay node is close to the sink there is more traffic to be forwarded than for that of the relay nodes far from the sink. For more energy efficient transmission this node can use short range transmission according to the relationship between optimal range and loaded traffic that we have deduced above for the transport network. However, nodes far from the sink have less data to forward and have longer idle times therefore they should use a longer radio range such that nodes not involved in routing can be put into the sleep state. Thus we are led to consider a non-uniform grid covering the network.

In this section we consider a sensor network collecting data at all nodes and forwarding all the data to a base station. We will compare the normal uniform grid of the GAF protocol with non-uniform grids where the grid size is adjusted according to the local traffic level. We consider a linear network where the density of nodes is uniform. The network contains a single sink on one edge at $x=0$. If each node produces $a$ Erlang of data then the traffic to be forwarded at a point that is $x$ meters away from base station is

$$
A(x)=(d-x) n_{d} a
$$

where $d$ is the size of the network and $n_{d}$ is the node density. Figure 3 shows a network that is covered by such a virtual grid.

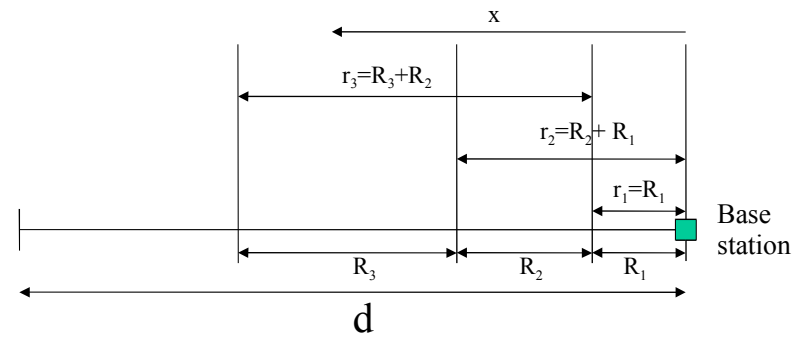

Figure 3: Linear network divided by virtual grids of different size

We consider two heuristic algorithms based on the range-traffic relationship (9). In the first algorithm, the grid sizes for grid section-specific traffic levels are calculated iteratively as follows

$R_{1}=R_{\text {opt }}\left(x=R_{1}\right), R_{2}=R_{\text {opt }}\left(x=R_{2}+R_{1}\right), \cdots, R_{i}=R_{o p t}\left(x=\sum_{j=1}^{i} R_{j}\right)$

where $R_{\text {opt }}$ is the optimal grid size for the regular transport network derived in section 2, equation (9). To guarantee any node in one grid section can connect to any node in the immediately adjacent downstream grid section, the radio range in each section has been chosen as follows

$$
r_{1}=R_{1}, r_{2}=R_{2}+R_{1}, \cdots_{i}, r_{i}=R_{i-1}
$$

In the case where the length of the linear network is $d=600 \mathrm{~m}$, node density $n_{d}=1 / 6$ per meter and every node produces data at a rate $a=0.003$ Erlang, the energy efficient optimal grid sizes are shown in table 1 .

\begin{tabular}{|l|l|}
\hline$i$ & $\mathrm{R}_{\mathrm{i}}(\mathrm{m})$ \\
\hline 1 & 20.8 \\
\hline 2 & 21.2 \\
\hline 3 & 21.6 \\
\hline 4 & 22.0 \\
\hline 5 & 22.5 \\
\hline
\end{tabular}




\begin{tabular}{|l|l|}
\hline 6 & 23.1 \\
\hline 7 & 23.7 \\
\hline 8 & 24.4 \\
\hline 9 & 25.1 \\
\hline 10 & 26.0 \\
\hline 11 & 27.0 \\
\hline 12 & 28.2 \\
\hline 13 & 29.6 \\
\hline 14 & 31.4 \\
\hline 15 & 33.7 \\
\hline 16 & 37.0 \\
\hline 17 & 42.1 \\
\hline 18 & 53.7 \\
\hline 19 & 86.7 \\
\hline
\end{tabular}

Table 1. Grid sizes calculated according to the algorithm of equations (11) for a $600 \mathrm{~m}$ linear network.

The traffic originating in section $i$ of the grid is forwarded to the base station by the relay node in section i-1 of the grid. The traffic handled by the routing node in any given section of the grid is passed directly to the routing node in the next section. The total power consumption due to receiving bits in the i'th grid section is given by

$$
P_{r}(i)=e_{r}\left(d-R_{1}-\ldots-R_{i}\right) n_{d} a B
$$

Recall that the transmitted traffic in the ith grid, $A_{t}(i)=\left(d-R_{1}-\ldots-R_{i}\right) n_{d} a$, is different to the received traffic, $A_{t}(i)=\left(d-R_{l^{-}} \ldots-R_{i-1}\right) n_{d} a$, and so the power consumed by transmission in the ith grid is

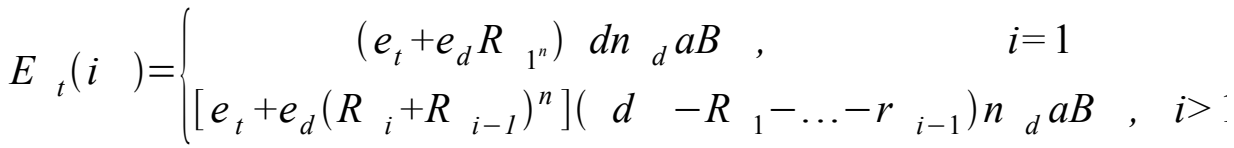
is

The energy consumption of the relay node during the fractional idle time $T_{\text {idle }}(i)=1-2\left(d-R_{1}-\ldots-R_{i}\right) n_{d} a$

$$
P_{i d l e}(i)=e_{r}\left[1-2\left(\begin{array}{lll}
d & \left.-R_{1}-\ldots-R_{i}\right) n_{d} a
\end{array}\right] B\right.
$$

Here we suppose the energy consumption in the idle state is the same as in the receiving state. The total power consumption of the whole network is

$$
P=\sum_{i=1}^{k} \quad P_{r}(i) \quad+P_{t}(i) \quad+P_{i d l e}(i)
$$

For the specific case represented by the network grid in table 1 we find the total power consumption to be, $P=9.51 \times 10^{-4} \mathrm{~J} / \mathrm{s}$.

However, we can improve still further on the total power consumption since the radio range $r_{i}=R_{i}+R_{i+1}$, which we actually use in the i'th grid, is not quite the same as the optimal range assumed in calculating the grid size $\left(r_{i}=2 R_{i}\right.$, see equation (9)). To save a little more power we should choose the optimal radio range instead of optimal grid size as we have done. The grid sizes and radio ranges can then be calculated as follows

$$
\begin{aligned}
& R_{1}+R_{2}=r_{\text {opt }}\left(x=R_{1}+R_{2}\right), \\
& R_{2}+R_{3}=r_{\text {opt }}\left(x=R_{1}+R_{2}+R_{3}\right), \cdots, R_{i-1}+R_{i}=r_{o p t}\left(x=\sum_{j=1}^{i} R_{j}\right)
\end{aligned}
$$

The first grid, $R_{l}$, is no longer specified by the algorithm (this is due to a boundary condition effect so that the radio range required in the first and second grid sections is determined by identical equations) and it can be chosen from the range $0 \leq R_{1} \leq\left[R_{1}+R_{2} \equiv r_{\text {opt }}\left(x=R_{1}+R_{2}\right)\right]$. The energy consumption of the whole network is almost invariant to the value of $R_{1}$ when $R_{1}$ is chosen from a large region around $r_{\text {opt }}\left(x=R_{1}+R_{2}\right) / 2$. To ensure the grid sizes change evenly and also that there is a high probability of finding a node in the grids we adopt $R_{1}=r_{\text {opt }}\left(x=R_{1}+R_{2}\right) / 2$. The grid sizes calculated according to algorithm 2 are shown in table 2 (note that the number of grids is one fewer in this case).

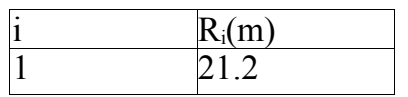




\begin{tabular}{|l|l|}
\hline 2 & 21.2 \\
\hline 3 & 22.0 \\
\hline 4 & 22.1 \\
\hline 5 & 23.1 \\
\hline 6 & 23.2 \\
\hline 7 & 24.3 \\
\hline 8 & 24.6 \\
\hline 9 & 25.9 \\
\hline 10 & 26.4 \\
\hline 11 & 28.0 \\
\hline 12 & 28.8 \\
\hline 13 & 31.1 \\
\hline 14 & 32.7 \\
\hline 15 & 36.5 \\
\hline 16 & 40.6 \\
\hline 17 & 52.2 \\
\hline 18 & 116 \\
\hline
\end{tabular}

Table 2. Grid sizes according to the algorithm of equations (17)

The total energy consumption is now $P=9.01 \times 10^{-4} \mathrm{~J} / \mathrm{s}$ which is slightly better than the result we obtained for algorithm 1 .

We now compare our range adjustment GAF algorithm with the unmodified GAF protocol where a uniform grid solution is used as shown in Figure 4.

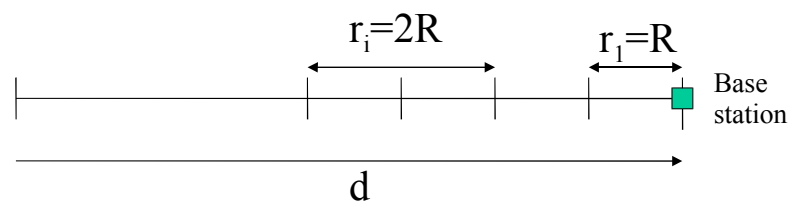

Figure 4: Linear network divided by equal size virtual grids

The energy consumption in the transmission, receiving and idle states respectively are

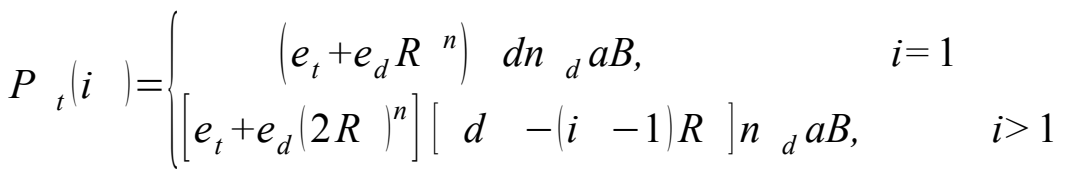

$$
\begin{aligned}
& P_{r}(i)=e_{r}(d-i R) n_{d} a B \\
& P_{\text {idle }}(i)=e_{r}\left[1-2\left(\begin{array}{ll}
d & -i R){ }_{d} a
\end{array}\right] B\right.
\end{aligned}
$$

The grid size $R$ is undetermined and is chosen from $0<R \leq d$. To obtain a more energy efficient GAF protocol, $R$ should be determined by some other methods. In Figure 5, the total energy consumption is plotted versus the number of grids for equal grid division. The energy consumption for the unequal grid division is also shown in Figure 5 as a single point since the number of grid sections is no longer a free parameter. We see that lower energy consumption has been achieved when the linear network is divided into a virtual routing grid according to the new range-traffic relationship we have proposed rather than using equal grid division. In our specific case, $50 \%$ of the total power is saved compared with the best case of equal grid division where the total energy consumption is $2.1 \times 10^{3} \mathrm{~J} / \mathrm{s}$. 


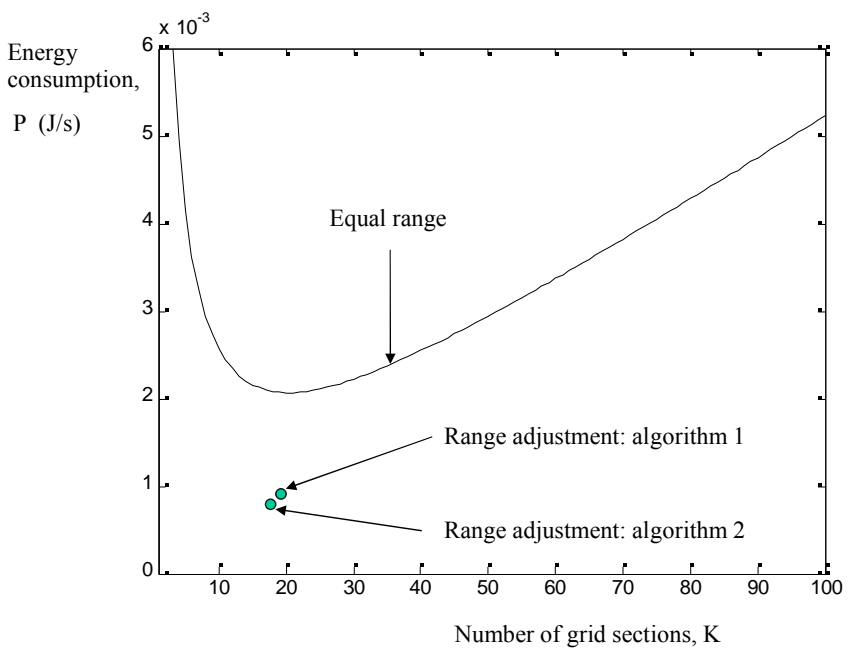

Figure 5: Total energy consumption of the network for the standard uniform grid and the new non-uniform grid.

\section{Conclusions}

In wireless ad hoc sensor networks, energy use is in many cases the most important constraint since it corresponds directly to operational lifetime. Topology management such as GAF puts the nodes not involved in forwarding to sleep to save energy. The radio range will affect the number of neighbour nodes, which collaborate to forward data to the sink. In this paper we have deduced the relationship between optimal radio range and traffic for a one dimensional network and find that half of the power can be saved, if the radio range is adjusted, compared with the best case where equal radio ranges are used. This would translate into a doubling of the network lifetime. We also showed that dividing the network into unequal grids according to the optimal range-traffic relationship can save a little more energy than by using the optimal size-traffic relationship. The concept of radio range adjustment can clearly be applied in other topology management algorithms to save energy.

\section{Acknowledgements}

The authors would lime to thank BT for financial support of this work.

\section{References}

[1] M. Stemm, R. H. Katz, Measuring and reducing energy consumption of network interfaces in hand-held devices, IEICE Transactions on Communications E80-B (8) (1997) pp. 1125-1131.

[2] O. Kasten, Energy consumption, Eth-Zurich, Swiss Federal Institute of Technology, 2001. Available from $<$ http://www.inf.ethz.ch/ kasten/research/bathtub/energy_consumption.html $>$.

[3] B. J. Chen, K. Jamieson, H. Balakrishnan, R. Morris, Span: an energy-efficient coordination algorithm for topology maintenance in ad hoc wireless networks, Wireless Networks 8 (5) (2002) 481-494.

[4] M. Bhardwaj, T. Garnett, A. P. Chandrakasan, Upper bounds on the lifetime of sensor networks, in: Proceedings of ICC'01, vol. 3, Helsinki, Finland, June 2001, pp. 785-790.

[5] R. Min, M. Bhardwaj, N. Ickes, A. Wang, A. Chandrakasan, The hardware and the network: total-system strategies for power aware wireless microsensors, in: Proceedings of IEEE CAS Workshop on Wireless Communications and Networking, Pasadena, USA, September 2002, pp. 36-12.

[6] W. R. Heinzelman, A. C., H. Balakrishnan, Energy-efficient communication protocol for wireless microsensor networks, in: Proceedings of HICSS'00, vol. 2, Hawaii, USA, January 2000, pp. 4-7.

[7] Y. Xu, J. Heidemann, D. Estrin, Geography-informed energy conservation for ad hoc routing, in: Proceedings of ACMMobile'01, Rome, Italy, July 2001, pp. 70-84. 
[8] A. Cerpa, D. Estrin, ASCENT: adaptive self-configuring sensor networks topologies, in: Proceedings of INFOCOM'02, vol. 3, New York, USA, June2002, pp. 1278-1287.

[9] Y. Xu, J. Heidemann, D. Estrin, Energy conservation by adaptive clustering for ad-hoc networks, in: Poster Session of MobiHoc'02, Lausanne, Switzerland, June 2002, pp. 255-263.

[10] Y. Xu., J. Heidemann, D. Estrin, Adaptive energy-conserving routing for multihop ad hoc networks, USC/ISI Research Report TR-2000-527, 2000. Available from <http://www.isi.edu/ johnh/PAPERS/Xu00a.html $>$.

[11] T. Rappaport, Wireless Communications: Principles \& Practice, Prentice-Hall Inc., New Jersey, 1996. 